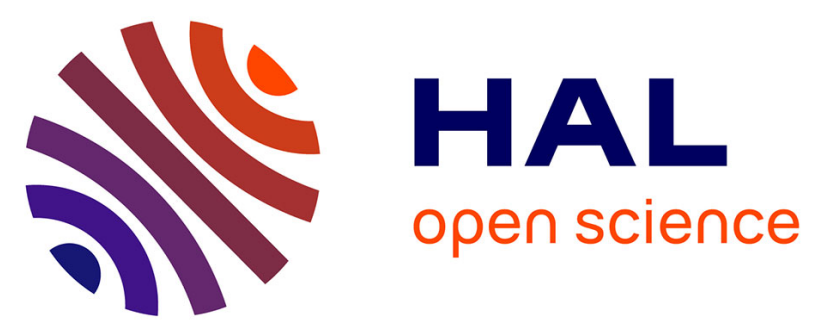

\title{
The association of theory of mind with language and visuospatial abilities in amyotrophic lateral sclerosis: a pilot study
}

Niki Panopoulou, Foteini Christidi, Panagiotis Kourtesis, Panagiotis

Ferentinos, Panagiota Karampetsou, Georgios Tsirtsiridis, Thomas

Theodosiou, Sofia Xirou, Vasiliki Zouvelou, Ioannis Evdokimidis, et al.

\section{To cite this version:}

Niki Panopoulou, Foteini Christidi, Panagiotis Kourtesis, Panagiotis Ferentinos, Panagiota Karampetsou, et al.. The association of theory of mind with language and visuospatial abilities in amyotrophic lateral sclerosis: a pilot study. Amyotrophic Lateral Sclerosis and Frontotemporal Degeneration, 2021, pp.1-8. 10.1080/21678421.2021.2013893 . hal-03481504

\author{
HAL Id: hal-03481504 \\ https://hal.inria.fr/hal-03481504
}

Submitted on 17 Dec 2021

HAL is a multi-disciplinary open access archive for the deposit and dissemination of scientific research documents, whether they are published or not. The documents may come from teaching and research institutions in France or abroad, or from public or private research centers.
L'archive ouverte pluridisciplinaire HAL, est destinée au dépôt et à la diffusion de documents scientifiques de niveau recherche, publiés ou non, émanant des établissements d'enseignement et de recherche français ou étrangers, des laboratoires publics ou privés. 


\section{The association of Theory of Mind with Language and Visuospatial Abilities in Amyotrophic Lateral Sclerosis: A Pilot Study}

Niki Panopoulou ${ }^{\mathrm{a}^{*}}$, Foteini Christidi ${ }^{\mathrm{a}, \mathrm{b}, \mathrm{c,d} \mathrm{d}^{*},}$ Panagiotis Kourtesis ${ }^{\mathrm{e}, \mathrm{f,g}, \mathrm{h}}$, Panagiotis Ferentinos ${ }^{\mathrm{c}}$, Panagiota Karampetsou ${ }^{\mathrm{a}}$, Georgios Tsirtsiridis ${ }^{\mathrm{a}}$, Thomas Theodosiou ${ }^{\mathrm{a}}$, Sofia Xirou ${ }^{\mathrm{b}}$, Vasiliki Zouvelou ${ }^{\mathrm{b}}$, Ioannis Evdokimidis ${ }^{\mathrm{b}}$, Michail Rentzos ${ }^{\mathrm{b}}$, Ioannis Zalonis ${ }^{\mathrm{a}}$

a Neuropsychological Lab, First Department of Neurology, Aeginition Hospital, Medical School, National and Kapodistrian University of Athens, Athens, Greece b First Department of Neurology, Aeginition Hospital, Medical School, National and Kapodistrian University of Athens, Athens, Greece

c Second Department of Psychiatry, Attikon General University Hospital, Medical School, National and Kapodistrian University of Athens, Athens, Greece d Laboratory of Medical Physics, Medical School, National and Kapodistrian University of Athens, Athens, Greece e National Research Institute of Computer Science and Automation, INRIA, Rennes, France

f Univ Rennes, Rennes, France

$g$ Research Institute of Computer Science and Random Systems, IRISA, Rennes, France $h$ French National Centre for Scientific Research, CNRS, Rennes, France.

*shared first-authorship, equal contribution

Corresponding author: $\quad$ Foteini Christidi

First Department of Neurology, Aeginition Hospital 72-74 Vas. Sofias Avenue, 11528 Athens, GR $+30-2107289141$

christidi.f.a@gmail.com 


\section{The association of Theory of Mind with Language and Visuospatial}

\section{Abilities in Amyotrophic Lateral Sclerosis: A Pilot Study}

Objective: Dysfunction of social cognition is well-recognized as one of amyotrophic lateral sclerosis (ALS) cognitive impairments. Previous studies have mostly associated social cognition subcomponents, including Theory of Mind (ToM), with executive dysfunction using highly demanding tasks. In the present study, we investigate dysfunction of affective ToM in a sample of ALS patients without dementia and evaluate any possible associations both with executive and non-executive dysfunction.

Method: We included 42 ALS patients and 30 healthy controls (HC) and administered the Edinburgh Cognitive and Behavioural Amyotrophic Lateral Sclerosis Screen (ECAS). Affective ToM was examined based on the ECAS judgment of preference task; total score and type of errors ("favourite", "unclassified") were recorded for all participants.

Results: A significant proportion of ALS patients (31\%) were impaired on ToM task, scoring significantly lower compared to HC. Impairments in ToM task were more frequent (45\%) in patients with cognitive impairment compared to those with intact cognition (15\%). ALS patients showed significantly more errors on ToM task compared to HC. A significant association was found between ToM score and ECAS language and visuospatial abilities but not fluency, executive or memory function.

Conclusion: Dysfunction of affective ToM appears prevalent in ALS patients without dementia, and associates with language and visuospatial abilities. These associations align with motor and extra-motor symptoms due to the degeneration across corresponding networks. Impaired ToM should be considered in clinical settings, since it might contribute to patients' social life, as well as the burden of their caregivers and relatives.

Keywords: Amyotrophic Lateral Sclerosis; Social Cognition; Theory of Mind; Language; Visuospatial 


\section{Introduction}

Cognitive and behavioural deficits are common in Amyotrophic Lateral Sclerosis (ALS), which are mostly related to frontotemporal dysfunction [1-2]. Although only 5$15 \%$ of patients meet the criteria for frontotemporal dementia (FTD) [3], approximately $50 \%$ or even more show cognitive impairments pertaining to executive functions and language, as well as behavioural changes $[4,5]$. The emerging cognitive, behavioural, and neuroimaging evidence have led to the proposal of the spectrum of ALS-FTD changes $[4,5]$. Recently, there has been an interest regarding potential impairments in social cognition in patients with ALS [6] and it has been shown that the social cognition is an inseparable part of the ALS-related cognitive profile [1].

A central part of the social cognition is the Theory of Mind (ToM). ToM refers to the understanding of emotions, desires and beliefs of others and the prevision of their future behaviour [7]. The cognitive ToM is relevant to the beliefs, thoughts and intentions of others [8-10]. The affective ToM is relevant to understanding the emotions of others [8-10]. Although ToM and social cognition deficits have been well-reported in both demented and non-demented patients with ALS [6,11], it remains poorly defined how these deficits are related to other cognitive processes and to patients' clinical characteristics.

Specifically, there are conflicting findings whether the social cognition may be attributed to executive dysfunction [12-13]. Several studies in non-demented ALS patients report significant correlation between executive functions and social cognition [13-15]. However, these studies used tasks with significant executive load such as the three subtests of the Happé's cartoon task, Written Scenarios tasks and the Reading the Mind in the Eyes task (RME) [13-15]. On the other hand, this association has also been 
reported when ToM was also examined using tasks with lower executive load, such as the Judgement of Preference task the False Belief task [16-18]. However, some studies found that deficits in ToM are not related to executive functions, while some of them found a significant correlation with long-term memory [19-20]. Also, studies reported a higher frequency of impaired social cognition in patients with a bulbar-onset $[14,18,20]$. The relationship of social cognition with executive functions and symptoms onset has not become clear due to methodological issues pertaining to the neuropsychological assessment.

The aim of the present study is to examine affective ToM in a sample of nondemented ALS patients and evaluate any possible associations with other cognitive functions and patients' clinical profile.

\section{Methods}

\section{Participants}

Forty-two non-demented ALS patients were included in the present study. All patients underwent clinical assessment, including full neurological and electrophysiological examination, and diagnosed with sporadic ALS according to the modified El Escorial diagnostic criteria for ALS [21]. For all patients, we calculated disease progression rate as (48 - ALSFRS-R) disease duration in months. Disease duration was calculated from first symptoms onset to scan date in months. Patients were subsequently referred for neuropsychological assessment as part of their routine clinical care. Also, 30 unrelated healthy volunteer participants comprised the healthy control (HC) group with similar demographic characteristics compared to ALS patients.

Inclusion criteria for the present study were the following: (a) absence of a history of other neurologic conditions affecting cognition (other than ALS for patients), 
(b) no serious psychiatric disease (e.g., major depression, schizophrenia), depression in ALS patients was assessed by the ALS-Depression Inventory (ADI-12) [22,23] (c) no family history of ALS, (d) absence of psychoactive drugs or other medication that could affect mental status, and (e) absence of dementia (Mini Mental State Examination score >24), preserved activities of daily living, preserved social and personal conduct and insight based on patients' and caregivers' self-reports; preserved discourse speech based on WAIS Vocabulary score and narrative speech .Patients' disability level was evaluated using the Revised Amyotrophic Lateral Sclerosis Functional Rating Scale (ALSFRS-R) [24]. All participants provided inform consent for the study, which was approved by the ethical committee of our institution.

\section{Material}

\section{Theory of Mind (ToM)}

The affective component of ToM was assessed using the Judgment of Preference task $[15,25]$, as it is included in the ECAS scale. This task assesses the ability to make a preference judgement based on eye gaze. It consists of a pre-experimental and an experimental condition; where there are six pictures, which one displays four objects of the same semantic category (e.g., animals, fruits, and furniture) positioned in the four corners. In the pre-experimental condition, the participants circle their favourite object from each picture. In the experimental condition, the participants circle the object which a face likes best. The correct answer is scored with 2 points. The errors are categorized as "favourite" (in which participants chose their personal favourite object instead; i.e., an egocentric response) and are scored with 0 points; or as "unclassified" which are scored with 1 point. For the purpose of the present study, the following scores were used: total score; number of "favourite" errors; number of "unclassified" errors. 


\section{Other Cognitive Functions}

All patients were examined using the Edinburgh Cognitive and Behavioural Amyotrophic Lateral Sclerosis Screen (ECAS) [26], adapted and validated for the Greek speaking population [27]. The Greek ECAS is able to identify cognitive and behavioural changes relevant to ALS [27], and it does not produce significant ceiling effects [28]. It assesses a range of functions typically affected in ALS (ALS-Specific: Fluency, Executive Functions, Language Functions) and not typically affected in ALS but are common in disorders of older adults (ALS Non-specific: Memory, Visuospatial Functions). An ECAS total score, as well as subscores for ALS-specific and ALS Nonspecific domains can be extracted. For the purpose of the present study, an ECAS Executive Functions score was also calculated without including the social cognition test. The ECAS also includes a separate semi-structured behavioural interview which yields a separate behavioural score and is undertaken with an informant/ caregiver separately from the patient and is based on the five key behavioural domains for diagnosing FTD using the most recent diagnostic criteria [12].

\section{Statistical analysis}

Assumptions for normality were initially evaluated and parametric statistics were then applied. Between-group differences on demographic characteristics were examined using t-test for independent samples (age, education) and chi-square (gender distribution). Between-group differences on ToM and ECAS scores were assessed using t-test for independent samples. Mixed factorial analysis of variance (ANOVA) was used to evaluate main effect and interaction regarding type of errors and diagnosis in two models. In the first model, diagnosis (i.e. ALS; HC) was used as between-subject factor and type of errors (i.e. "favourite"; "unclassified") was used as within-subject factor. 
For the second model, ALS patients were categorized as impaired and non-impaired based on the ECAS total score and a cut-off score of 2SD using available normative data [27]. Thus, three groups (i.e. impaired ALS; non-impaired ALS; HC) were considered in the diagnosis which was used as the between-subject factor while type of errors was the within-subject factor. Post-hoc Bonferroni comparisons between impaired ALS, non-impaired ALS and HC were applied. Correlation analyses between ToM total score and ECAS subscores (ECAS Language; ECAS Verbal Fluency; ECAS Executive Functions (without ECAS social cognition subtest); ECAS Memory; ECAS Visuospatial Functions), as well as clinical variables (disease duration, ALSFRS-R, progression rate) was conducted using Pearson's r. Comparisons on ToM total score between peripheral- and spinal-onset ALS patients was conducted using t-test for independent samples. The level of statistical threshold was set at $p<0.05$ and all analyses were conducted using IBM SPSS 20.

\section{Results}

Table 1 displays the descriptive statistics for ALS and HC groups.

Table 1. Descriptive Statistics of ALS patients and Healthy Controls

\begin{tabular}{cccc}
\hline & ALS & HC & p-value \\
\hline Age (yrs) & $60.57(9.90)$ & $60.67(7.36)$ & 0.965 \\
Education (yrs) & $10.55(3.68)$ & $11.97(3.42)$ & 0.102 \\
Gender (M/F) & $24 / 18$ & $13 / 17$ & 0.255 \\
ALSFRS-R & $39.83(5.377)$ & - & - \\
Disease duration (m) & $22.11(32.36)$ & - & - \\
Progression rate & $0.57(0.82)$ & - & - \\
Cognition (P / Imp) & $21 / 21$ & $30 / 0$ & $<0.001$ \\
ECAS total score & $89.55(20.55)$ & $106.53(9.77)$ & $<0.001$ \\
ECAS ALS-specific score & $64.98(16.97)$ & $78.23(7.68)$ & $<0.001$ \\
ECAS ALS-nonspecific score & $24.57(5.55)$ & $28.30(3.23)$ & 0.002 \\
ECAS Behavioural score & $1.21(1.28)$ & $0.0(0.0)$ & $<0.001$ \\
\hline
\end{tabular}


Note. $H C=$ healthy controls; yrs $=$ years $; m=$ months $M / F=$ male $/$ female $;$ ALSFRS $-R=$ revised Amyotrophic Lateral Sclerosis Functional Rating Scale; P / Imp = Preserved / Impaired; ECAS = Edinburgh Cognitive and Behavioural ALS Screen.

\section{Between-group differences on ToM}

ALS patients scored significantly worse $(p=0.005 ;|d|=0.69)$. The $31 \%$ of patients scored in the impaired range. Also, $45 \%$ of ALS patients with impaired cognition showed impaired ToM while $15 \%$ of ALS patients with preserved cognition showed impaired ToM.

Significant main effects of diagnosis $(p=0.006 ; \eta 2=0.103)$ and type of errors (i.e., "favourite" and "unclassified"; $F=13.764 ; p<0.001 ; \eta 2=0.164$ ) were found. The interaction effect between them was not significant $(p=0.762 ; \eta 2=0.001)$. ALS patients made significantly more "favourite" $(\mathrm{p}=0.032 ;|\mathrm{d}|=0.52)$ and "unclassified" $(\mathrm{p}=0.042 ;|\mathrm{d}|=0.50)$ errors compared to HC (see Figure 1 and Figure 2$)$; yet, both $\operatorname{ALS}(\mathrm{p}=0.007 ;|\mathrm{d}|=0.56)$ and $\mathrm{HC}(\mathrm{p}<.001 ;|\mathrm{d}|=0.80)$ made significantly more "unclassified" errors compared to the "favourite" ones.

Figure 1. "Favourite" Errors in ALS and Healthy Controls

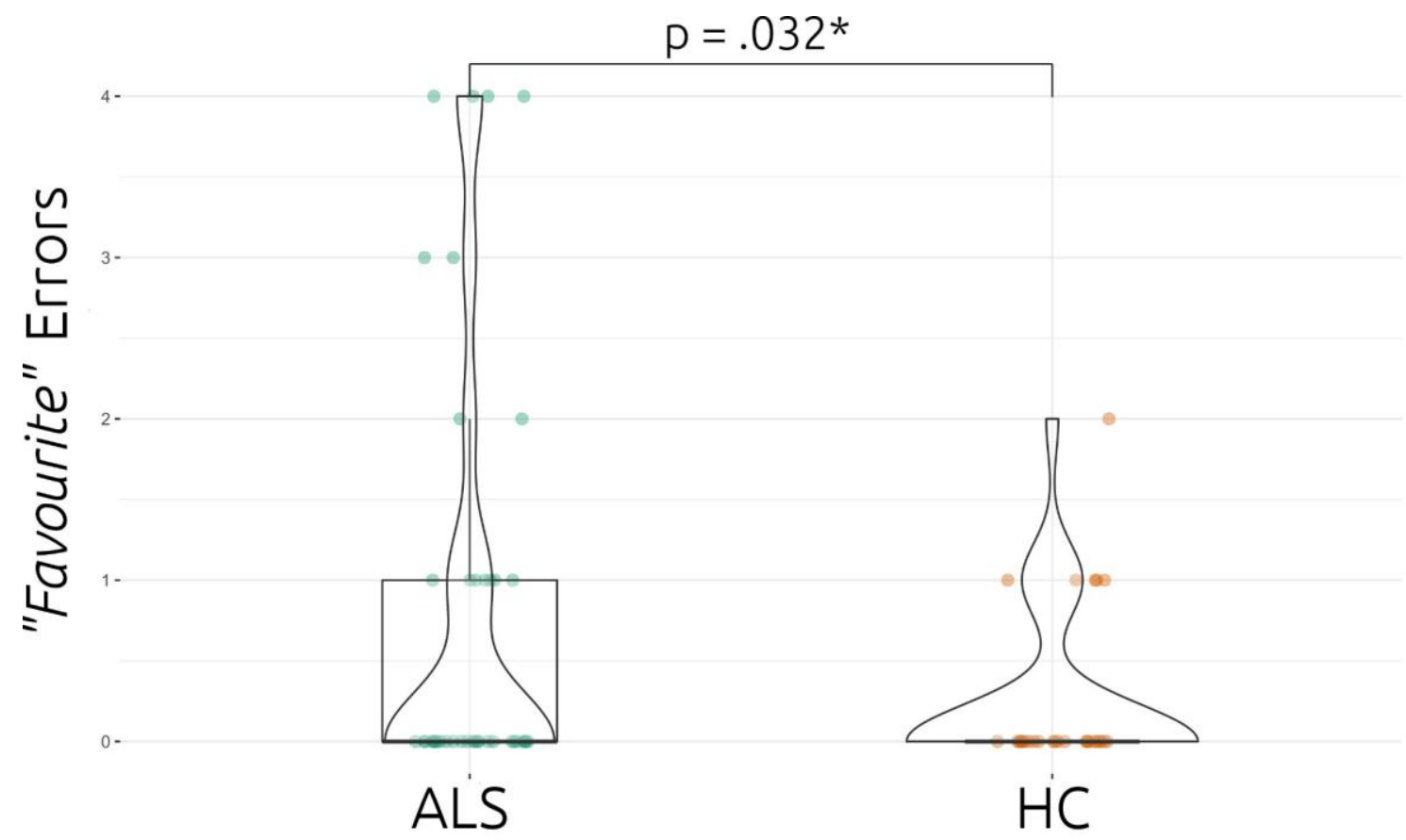


Figure 2. "Unclassified" Errors in ALS and Healthy Controls.

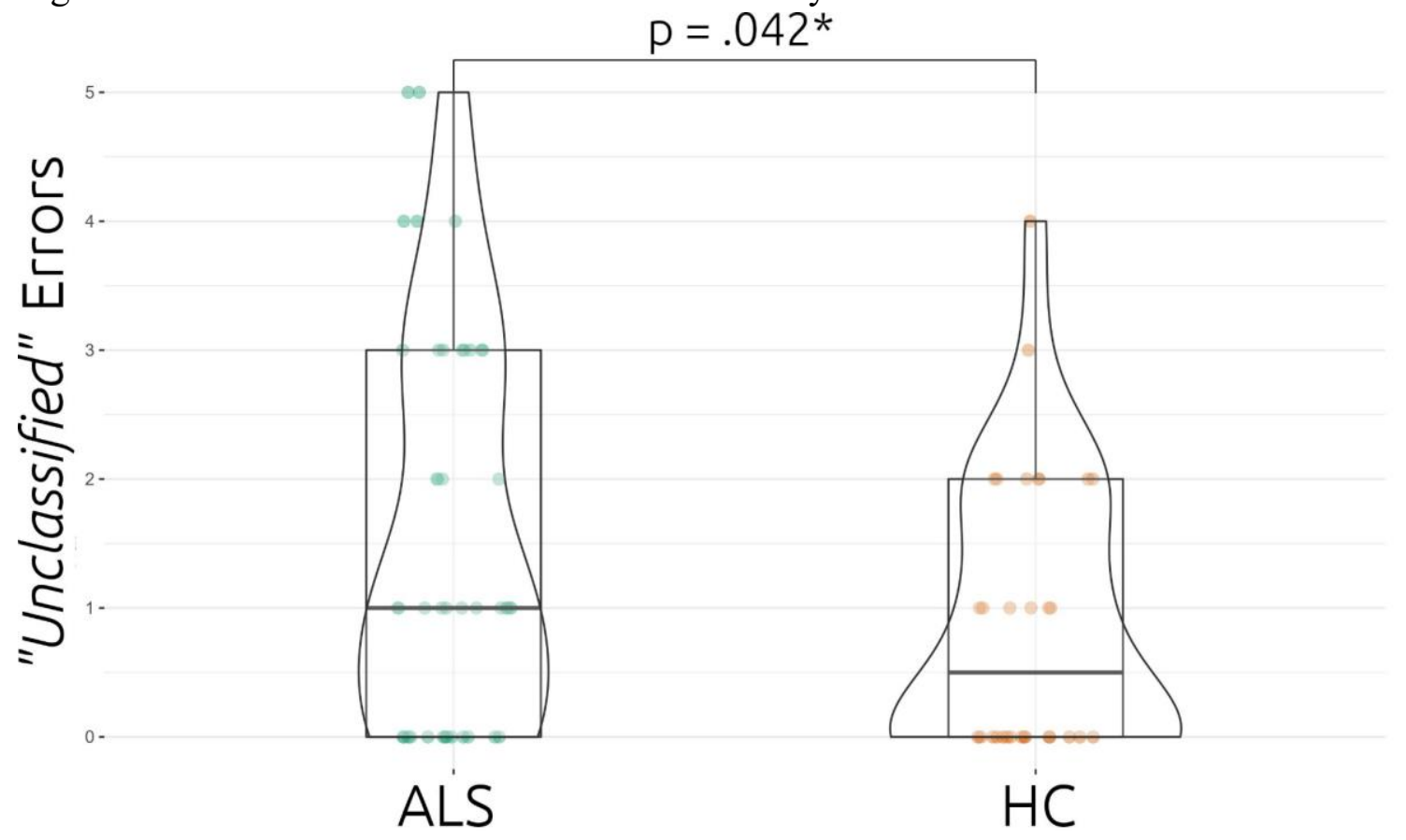

Significant differences in social cognition total score $(F=8.600 ; p<0.001 ; \eta 2=$ $0.200)$ and number of total errors $(F=8.086 ; p<0.001 ; \eta 2=0.190)$ were revealed. Impaired ALS patients performed significantly worse compared to non-impaired ALS patients $(\mathrm{p}=0.018)$ and $\mathrm{HC}(\mathrm{p}<0.001$; see Figure 3$)$. No other significant difference between the groups was found.

Figure 3. Theory of Mind Score per Diagnosis

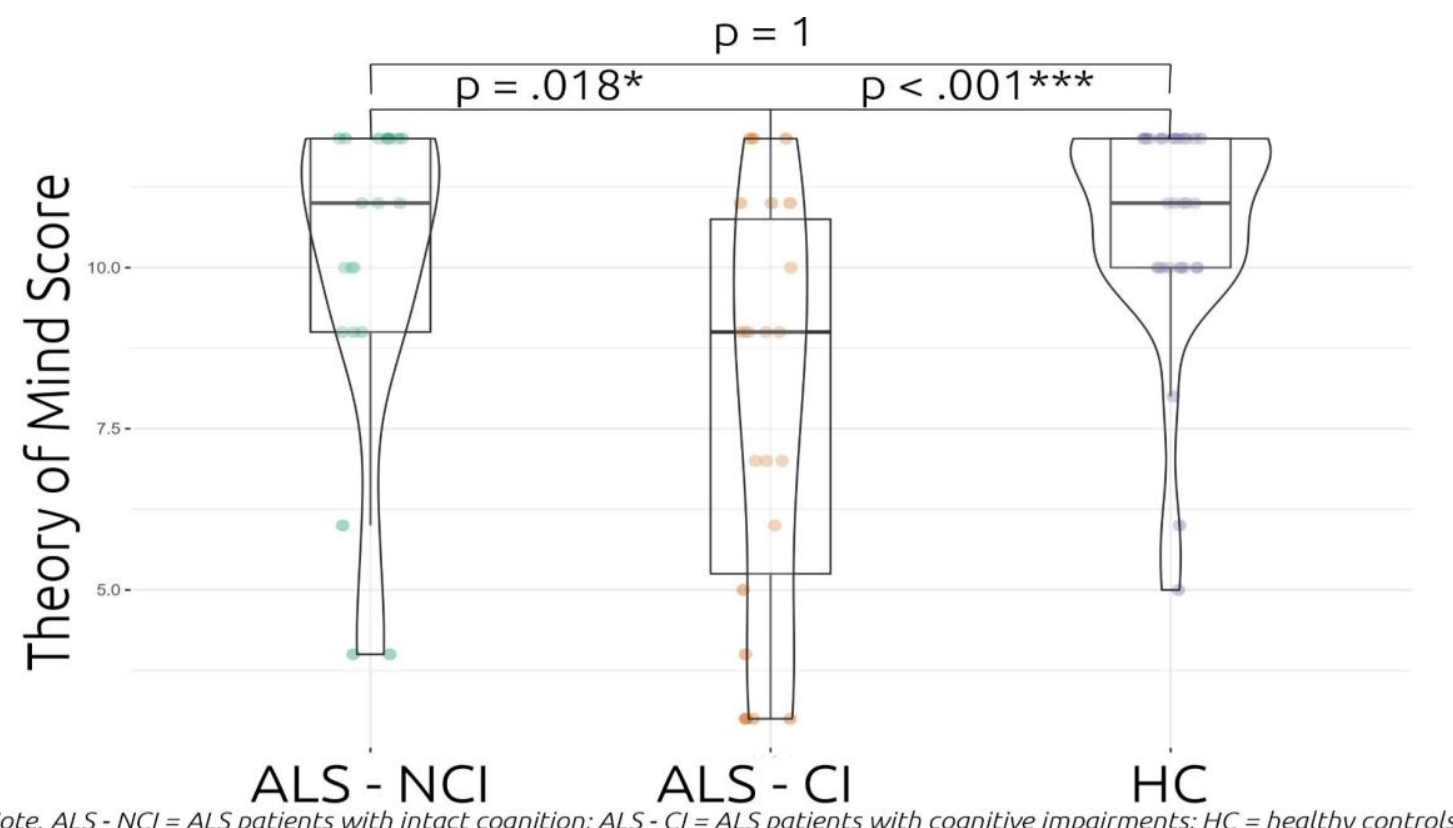

Note. $A L S-N C I=A L S$ patients with intact cognition; $A L S-C l=A L S$ patients with cognitive impairments; $H C=$ healthy controls 
Significant main effects of diagnosis (HC; impaired ALS; non-impaired ALS) $(\mathrm{p}<$ $0.001 ; \eta 2=0.190)$ and type of errors $(p<0.001 ; \eta 2=0.172)$ were detected, while the interaction effect was non-significant $(p=0.954 ; \eta 2=0.001)$. Post-hoc comparisons revealed that ALS patients with cognitive impairments made significantly more "unclassified" ( $p=0.027$; see Figure 4) and "favourite" ( $p=0.008$; see Figure 5) errors compared to $\mathrm{HC}$, while the rest of differences were not significant.

Figure 4. "Unclassified” Errors per Diagnosis

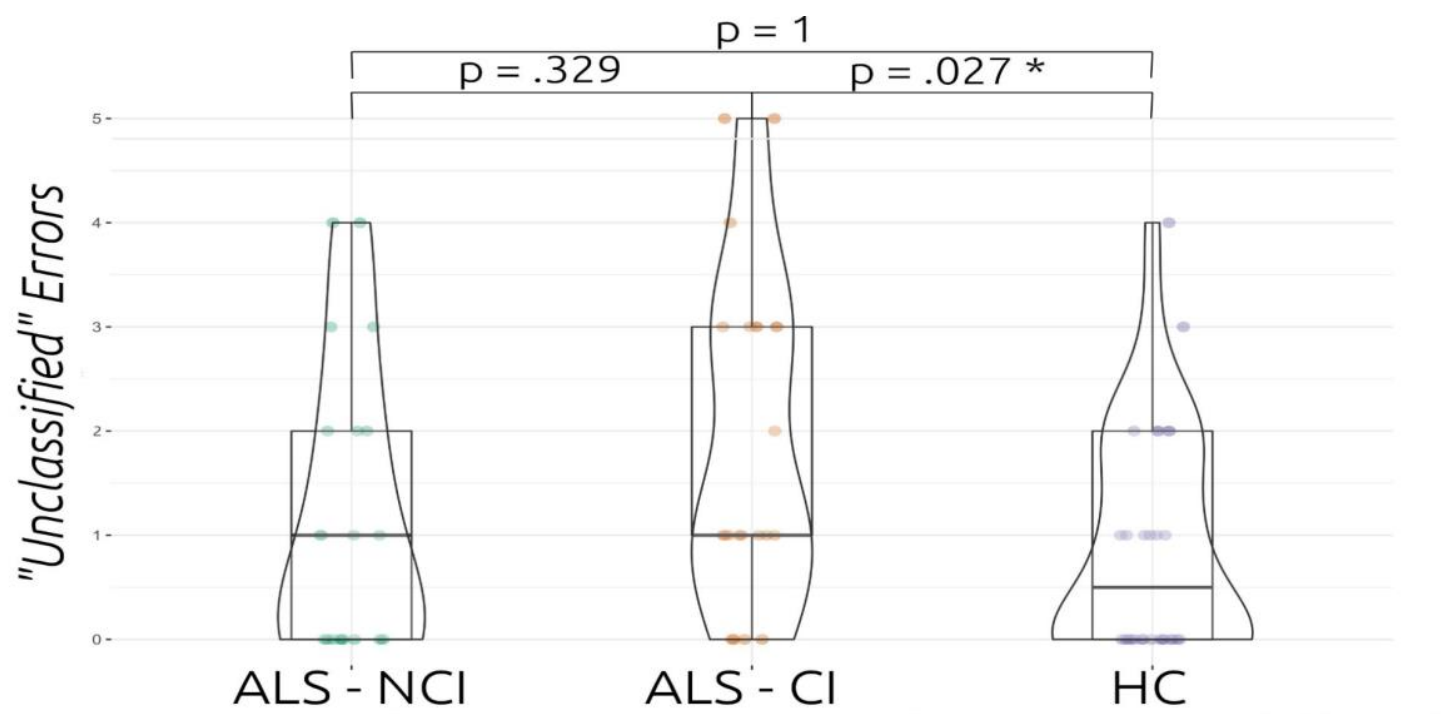

Note. ALS - NCI = ALS patients with intact cognition; ALS - CI = ALS patients with cognitive impairments; HC = healthy controls

Figure 5. "Favourite" Errors per Diagnosis

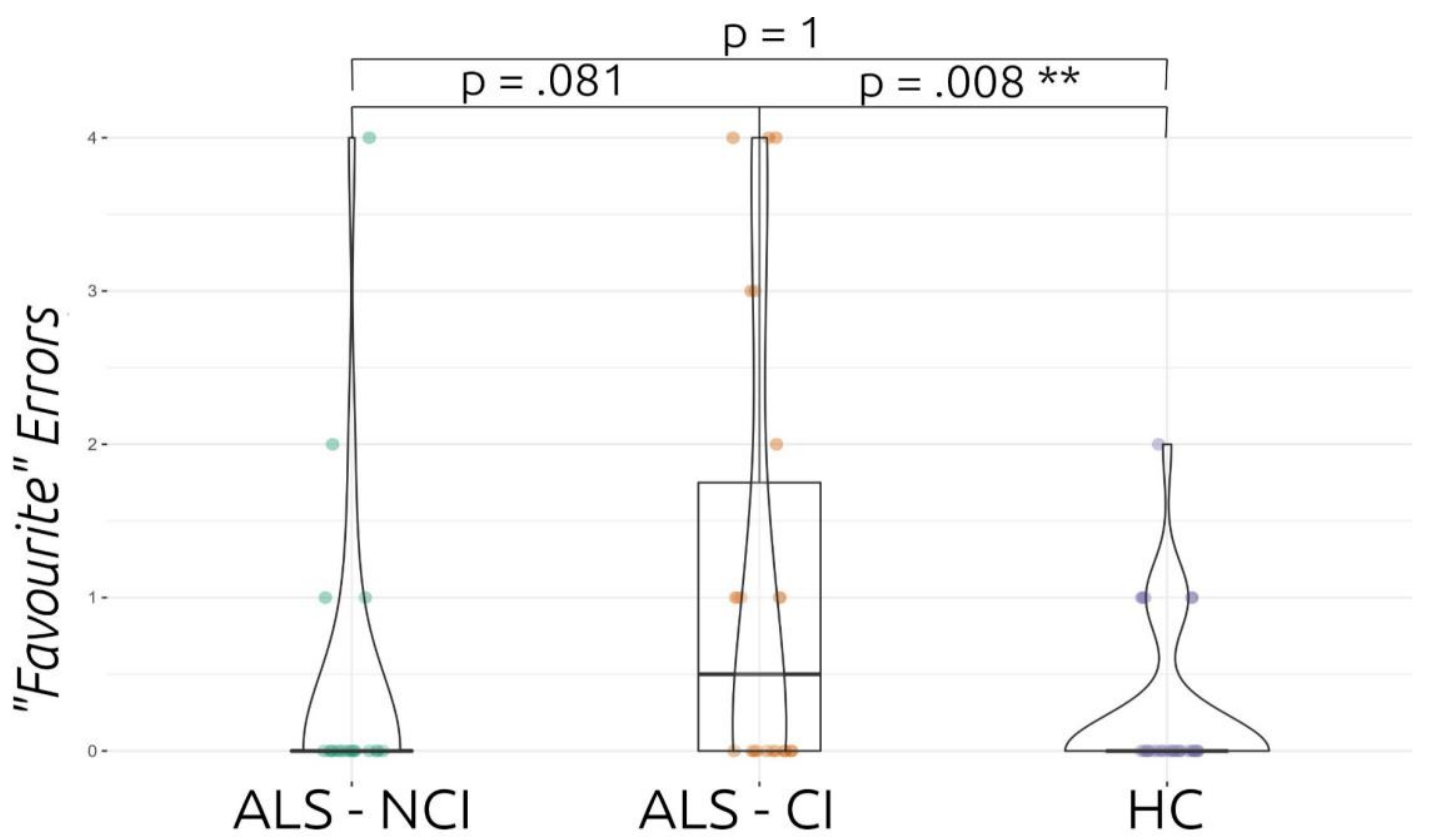

Note. $A L S-N C I=A L S$ patients with intact cognition; $A L S-C l=A L S$ patients with cognitive impairments; $H C=$ healthy controls 


\section{Correlations between ToM and ALS Clinical profile}

ECAS Language $(r=0.047 ; p=0.003)$ and ECAS Visuospatial Functions $(r=0.455 ; p$ $=0.002)$ were significantly correlated with the social cognition total score. However, no association were found between social cognition total score and ECAS Executive Functions $(r=0.221 ; p=0.159)$, ECAS Verbal Fluency $(r=0.175 ; p=0.273)$ or ECAS memory $(\mathrm{r}=0.184 ; \mathrm{p}=0.249)$.

Social cognition was significantly associated with the performance on language tasks. The ToM score was found to be substantially associated with ECAS Naming $(r=$ 0.435; $\mathrm{p}=0.002)$, ECAS Comprehension $(\mathrm{r}=0.343 ; \mathrm{p}=0.013)$, and ECAS Spelling $(\mathrm{r}$ $=0.300 ; \mathrm{p}=0.028)$. Also, a significant correlation was detected between the ToM score and the ECAS Sentence Completion (i.e., executive function task; $r=0.343 ; p=0.013$ ). Finally, the ToM score was correlated with the ECAS Cubes (i.e., visuospatial task; $r=$ $0.413 ; \mathrm{p}=0.003)$.

The "favourite" errors showed significant negative correlation with the ECAS Naming $(r=-0.437 ; p=0.002)$, ECAS Comprehension $(r=-0.446 ; p=0.002)$, ECAS Sentence Completion (i.e., executive function task; $r=-0.287 ; p=0.034$ ), and ECAS Cubes (i.e., visuospatial task; $r=-0.288 ; p=0.032$ ). In contrast, the "unclassified" errors were substantially negatively associated only with the ECAS Spelling ( $\mathrm{r}=$ $0.398 ; \mathrm{p}=0.005)$ and the ECAS Cubes $(\mathrm{r}=-0.340 ; \mathrm{p}=0.014)$.

Finally, the ToM score showed substantial negative correlations with the behavioural disinhibition item (i.e., loss of manners; $r=-0.384 ; p=0.031$ ) and the ECAS Psychosis score $(r=-0.384 ; \mathrm{p}=0.031)$. Notably, the "favourite" errors showed robust positive correlations with behavioural disinhibition - loss of manners $(r=0.456$; $\mathrm{p}=0.002)$ and the ECAS Psychosis score $(\mathrm{r}=0.456 ; \mathrm{p}=0.002)$, while the 
"unclassified" errors were not correlated with any behavioural change. Also, there was no significant correlation between social cognition total score and patients' clinical parameters (disease duration, ALSFRS-R, progression rate). We did not find any significant difference between peripheral and bulbar onset subgroups.

\section{Discussion}

The present study provided further evidence regarding impaired affective ToM in a well-characterized group of non-demented ALS patients, revealing that approximately one out of three non-demented patients shows impaired affective ToM, as determined by eye gaze in Judgement of Preference task of the ECAS scale. In our group, this impaired performance was unrelated to patients' clinical status and was not driven by any executive dysfunction; yet, it was more evident in patients with impaired cognition in general and was predominantly associated with patients' language and visuospatial abilities.

\section{ToM and type of errors in ALS}

$31 \%$ of patients had impaired performance on affective ToM and ALS patients performed worse than HC. Our findings are in line with the amassed findings of recent meta-analysis and review papers $[1,6,11]$, which further signify the importance of measuring social cognition in ALS [5]. A significant proportion of cognitively impaired ALS patients were also impaired in ToM (45\% vs. $15 \%$ in cognitively non-impaired ALS patients). These findings postulate that impairments in ToM are prevalent in ALS patients with cognitive impairments.

ALS patients produced significantly more "unclassified" than "favourite" errors (a pattern which was also observed in HC) and made significantly more errors of both 
types compared to HC. This may suggest that our non-demented ALS patients did not have significant difficulties in inhibiting egocentric processing, a deficit which is prominent in patients with the behavioural variant of FTD (bvFTD). Notably, all patients complied with tasks instructions in pre-experimental task condition, which indicates that failures in the experimental condition are unlikely to be due to comprehension impairment.

Previously, non-demented ALS patients were found to make equal number of "favourite" and "unclassified" errors [18]. These findings may underlie difficulties in inhibiting egocentric processes and shifting attention, which might be associated with the executive component of social cognition [29]. FTD patients produce a higher number of "favourite" errors than patients with Huntington's disease, due to their impaired inhibition of egocentric impulses [15].

In our study, the ALS patients with cognitive impairments produced significantly more "favourite" errors than the healthy controls, while the ALS patients with intact cognition did not differed significantly from healthy controls. However, similar significant differences were also found in the production of "unclassified" errors. Nevertheless, the differences appear greater regarding the "favourite" errors, rather than the "unclassified" errors. Furthermore, while significant negative correlations were identified between ToM score and ECAS Psychosis score and Behavioural disinhibition item (i.e., loss of manners), only the "favourite" errors were found to be substantially correlated with them. Of note, these behavioural changes are prevalent in ALS-FTD [5], hence, the quantity of "favourite" errors may be an early indicator of FTD. Nevertheless, considering that the type of errors has not been thoroughly studied in ALS specifically without dementia, further investigation is definitely needed in larger sample sizes with additional neuroimaging techniques to 
identify the anatomical substrate of each type of errors.

\section{ToM and clinical features in $A L S$}

Previous studies identified a significant correlation between symptoms' onset and social cognition performance; patients with bulbar onset scored worse than patients with spinal onset as determined by the Reading in the Eye Task [20,30]. However, we did not find any association between patients' clinical status and their performance in ToM. This disagreement may be a result due to the substantial differences between the two tasks. In our study, the Judgment of Preference task examines the egocentric biases in recognising the preference of others. On the other hand, the Reading in the Eye Task pertains to reading facial features for recognising diverse emotional states of the others. This discrepancy hence may indicate that the recognition of emotional states may be related with the symptoms' onset, while the egocentric biases are not.

\section{ToM and other cognitive functions in ALS}

Recent studies indicate an association between social cognition and executive functions (e.g., $[13,14])$ and verbal fluency $[12,19]$ in non-demented ALS patients. However, other studies have failed to find such an association (e.g., [20]). In our study, performance on a ToM task with low executive demands was predominantly associated with language and visuospatial abilities, which may suggest the independence between executive abilities and ToM performance. Affective ToM involves medial prefrontal networks $[7,16]$ in contrast to executive dysfunction which is related to dorsolateral prefrontal networks [31]. ToM appears to mediate not from a single set of brain regions, but from a distributed brain network [32], where at least two white matter tracts in the right hemisphere may be involved in face-based mentalizing (i.e., a component of social cognition) [33]. 
In our study, the performance on ToM and the production of "favourite" errors were predominantly associated with the performance on several language tasks, as well as with the performance on a visuospatial task (i.e., cube counting) which requires three-dimensional perceptual processes. These findings highlight the involvement of language and three-dimensional visuospatial abilities in affective ToM). From a developmental point of view, social cognition is required for language acquisition, deduce word meanings and communicate pragmatically [34,35]. Also, Thakkar \& Park (2010) provide similar findings in healthy participants suggesting a common link between the ability of visuospatial transformation and the internal representations and interactions with the physical world [36]. In addition, the role of visuospatial abilities in ToM has been reported in patients with Parkinson's disease, supporting that visuospatial deficits are associated with patients' social interaction and activities in daily living [37]. ToM, language, and visuospatial abilities, appear to share common pathways due to evolutionary changes in brain regions connectivity [38-39]. Similarly, in ALS and ALSFTD, the study of evolutionary adaptations in brain connectivity [40], assists with interpreting motor and extra-motor deficits based on the progressive degeneration over interlinked networks [41-45].

\section{Limitations}

Limitations of the present study includes the evaluation of only one subcomponent of social cognition, which is the affective ToM requiring preference judgement based on eye gaze from a picture and inferences regarding one's emotions. Also, this task requires two-dimensional visuospatial abilities (i.e., following the eye gaze from a picture), which may have affected the associations between ToM and visuospatial performance. However, the ToM performance was only associated with the threedimensional task and not with the two-dimensional visuospatial tasks. Nevertheless, 
future studies should include more sophisticated measures to test the type of errors and also addresses the issue of impaired ToM and other social cognition components in experimental environments (such the ones related to neuropsychological evaluation) and patients' everyday life.

\section{Conclusion}

This study demonstrated evidence of affective ToM dysfunction in non-demented ALS patients, which was associated with language and visuospatial abilities. These associations possibly tap onto common evolutionary patterns of these cognitive functions, and align with the evolutionary perspective of motor and extra-motor symptoms due to the degeneration across corresponding networks. Our findings highlight the importance of impaired ToM that should be considered in clinical settings, since this might contribute to patients' social life, as well as the burden of their caregivers and relatives.

\section{References}

1.Beeldman E, Raaphorst J, Twennaar MK, de Visser M, Schmand BA, de Haan RJ. The cognitive profile of ALS: a systematic review and meta-analysis update. Journal of Neurology, Neurosurgery \& Psychiatry. 2016 Jun 1;87(6):611-9.

2.Goldstein LH, Abrahams S. Changes in cognition and behaviour in amyotrophic lateral sclerosis: nature of impairment and implications for assessment. The Lancet Neurology. 2013 Apr 1;12(4):368-80.

3.Rascovsky K, Hodges JR, Knopman D, Mendez MF, Kramer JH, Neuhaus J, Van Swieten JC, Seelaar H, Dopper EG, Onyike CU, Hillis AE. Sensitivity of revised diagnostic criteria for the behavioural variant of frontotemporal dementia. Brain. 2011 Sep 1;134(9):2456-77.

4.Strong MJ, Grace GM, Freedman M, Lomen-Hoerth C, Woolley S, Goldstein LH, Murphy J, Shoesmith C, Rosenfeld J, Leigh PN, Bruijn L. Consensus criteria for the diagnosis of frontotemporal cognitive and behavioural syndromes in amyotrophic lateral sclerosis. Amyotrophic Lateral Sclerosis. 2009 Jan $1 ; 10(3): 131-46$.

5.Strong MJ, Abrahams S, Goldstein LH, Woolley S, Mclaughlin P, Snowden J, Mioshi E, Roberts-South A, Benatar M, HortobáGyi T, Rosenfeld J. Amyotrophic lateral sclerosis-frontotemporal spectrum disorder (ALS-FTSD): Revised diagnostic criteria. Amyotrophic Lateral Sclerosis and Frontotemporal Degeneration. 2017 Apr 3;18(3-4):153-74. 
6.Bora E. Meta-analysis of social cognition in amyotrophic lateral sclerosis. Cortex. 2017 Mar 1;88:1-7.

7.Shamay-Tsoory SG, Aharon-Peretz J. Dissociable prefrontal networks for cognitive and affective theory of mind: a lesion study. Neuropsychologia. 2007 Jan 1;45(13):3054-67.

8.Coricelli G. Two-levels of mental states attribution: from automaticity to voluntariness. Neuropsychologia. 2005 Jan 1;43(2):294-300.

9.Abu-Akel A, Shamay-Tsoory S. Neuroanatomical and neurochemical bases of theory of mind. Neuropsychologia. 2011 Sep 1;49(11):2971-84.

10.Gallagher HL, Frith CD. Functional imaging of 'theory of mind'. Trends in Cognitive Sciences. 2003 Feb 1;7(2):77-83.

11.Christidi F, Migliaccio R, Santamaría-García H, Santangelo G, Trojsi F. Social Cognition Dysfunctions in Neurodegenerative Diseases: Neuroanatomical Correlates and Clinical Implications. Behavioural Neurology. 2018 Apr 26.

12.Meier SL, Charleston AJ, Tippett LJ. Cognitive and behavioural deficits associated with the orbitomedial prefrontal cortex in amyotrophic lateral sclerosis. Brain. 2010 Nov 1;133(11):3444-57.

13.Watermeyer TJ, Brown RG, Sidle KC, Oliver DJ, Allen C, Karlsson J, Ellis CM, Shaw CE, Al-Chalabi A, Goldstein LH. Executive dysfunction predicts social cognition impairment in amyotrophic lateral sclerosis. Journal of Neurology. 2015 Jul;262(7):1681-90.

14.Gibbons ZC, Snowden JS, Thompson JC, Happe F, Richardson A, Neary D. Inferring thought and action in motor neurone disease. Neuropsychologia. 2007 Jan 1;45(6):1196-207.

15.Snowden JS, Gibbons ZC, Blackshaw A, Doubleday E, Thompson J, Craufurd D, Foster J, Happé F, Neary D. Social cognition in frontotemporal dementia and Huntington's disease. Neuropsychologia. 2003 Jan 1;41(6):688-701.

16.Bertoux M, Volle E, De Souza LC, Funkiewiez A, Dubois B, Habert MO. Neural correlates of the mini-SEA (Social cognition and Emotional Assessment) in behavioral variant frontotemporal dementia. Brain Imaging and Behavior. 2014 Mar;8(1):1-6.

17.Carluer L, Mondou A, Buhour MS, Laisney M, Pélerin A, Eustache F, Viader F, Desgranges B. Neural substrate of cognitive theory of mind impairment in amyotrophic lateral sclerosis. Cortex. 2015 Apr 1;65:19-30.

18.Girardi A, MacPherson SE, Abrahams S. Deficits in emotional and social cognition in amyotrophic lateral sclerosis. Neuropsychology. 2011 Jan;25(1):53.

19.Cavallo M, Adenzato M, MacPherson SE, Karwig G, Enrici I, Abrahams S. Evidence of social understanding impairment in patients with amyotrophic lateral sclerosis. PLoS one. 2011 Oct 5;6(10):e25948.

20.Trojsi F, Siciliano M, Russo A, Passaniti C, Femiano C, Ferrantino T, De Liguoro S, Lavorgna L, Monsurrò MR, Tedeschi G, Santangelo G. Theory of mind and its neuropsychological and quality of life correlates in the early stages of amyotrophic lateral sclerosis. Frontiers in Psychology. 2016 Dec 12;7:1934.

21.Brooks BR, Miller RG, Swash M, Munsat TL. El Escorial revisited: revised criteria for the diagnosis of amyotrophic lateral sclerosis. Amyotrophic Lateral Sclerosis and other Motor Neuron Disorders. 2000 Jan 1;1(5):293-9.

22.Ferentinos P, Paparrigopoulos T, Rentzos M, Zouvelou V, Alexakis T, Evdokimidis I. Prevalence of major depression in ALS: comparison of a semi-structured interview and four self-report measures. Amyotrophic Lateral Sclerosis. $2011 \mathrm{Jul}$ 1;12(4):297-302. 
23.Hammer EM, Häcker S, Hautzinger M, Meyer TD, Kübler A. Validity of the ALSDepression-Inventory (ADI-12) - a new screening instrument for depressive disorders in patients with amyotrophic lateral sclerosis. Journal of Affective Disorders. 2008 Jul 1;109(1-2):213-9.

24.Gordon PH, Cheng B, Katz IB, Pinto M, Hays AP, Mitsumoto H, Rowland LP. The natural history of primary lateral sclerosis. Neurology. 2006 Mar 14;66(5):647-53.

25.Baron-Cohen S, Campbell R, Karmiloff-Smith A, Grant J, Walker J. Are children with autism blind to the mentalistic significance of the eyes?. British Journal of Developmental Psychology. 1995 Nov;13(4):379-98.

26.Abrahams S, Newton J, Niven E, Foley J, Bak TH. Screening for cognition and behaviour changes in ALS. Amyotrophic Lateral Sclerosis and Frontotemporal Degeneration. 2014 Mar 1;15(1-2):9-14.

27.Kourtesis P, Christidi F, Margioti E, Demenega C, Rentzos M, Evdokimidis I, Abrahams $\mathrm{S}$. The Edinburgh cognitive and behavioral amyotrophic lateral sclerosis screen (ECAS): sensitivity in differentiating between ALS and Alzheimer's disease in a Greek population. Amyotrophic Lateral Sclerosis and Frontotemporal Degeneration. 2020 Jan 2;21(1-2):78-85.

28.Kourtesis P, Margioti E, Demenega C, Christidi F, Abrahams S. A Comparison of the Greek ACE-III, M-ACE, ACE-R, MMSE, and ECAS in the Assessment and Identification of Alzheimer's Disease. Journal of the International Neuropsychological Society. 2020 Sep;26(8):825-34.

29.van der Hulst EJ, Bak TH, Abrahams S. Impaired affective and cognitive theory of mind and behavioural change in amyotrophic lateral sclerosis. Journal of Neurology, Neurosurgery \& Psychiatry. 2015 Nov 1;86(11):1208-15.

30.Burke T, Elamin M, Galvin M, Hardiman O, Pender N. Caregiver burden in amyotrophic lateral sclerosis: a cross-sectional investigation of predictors. Journal of Neurology. 2015 Jun;262(6):1526-32.

31.Roca, M., Torralva, T., Gleichgerrcht, E., Woolgar, A., Thompson, R., Duncan, J., \& Manes, F. (2011). The role of Area 10 (BA10) in human multitasking and in social cognition: a lesion study. Neuropsychologia, 49(13), 3525-3531.

32.Schaafsma SM, Pfaff DW, Spunt RP, Adolphs R. Deconstructing and reconstructing theory of mind. Trends in Cognitive Sciences. 2015 Feb 1;19(2):65-72.

33.Nakajima R ., Yordanova YN, Duffau H, Herbert G. Neuropsychological evidence for the crucial role of the right arcuate fasciculus in the face-based mentalizing network: A disconnection analysis, Neurophycologia, 2018; (18)30024-1.

34.Clark EV. The principle of contrast: A constraint on language acquisition. In $B$. MacWhinney (Ed.), Mechanisms of language acquisition, Lawrence Erlbaum Associates, Inc. 1987:1-33.

35.Macnamara J. Cognitive basis of language learning in infants. Psychological Review. 1972 Jan;79(1):1.

36.Thakkar KN, Park S. Empathy, schizotypy, and visuospatial transformations. Cognitive Neuropsychiatry. 2010 Sep 1;15(5):477-500.

37.McKinlay A, Albicini M, Kavanagh PS. The effect of cognitive status and visuospatial performance on affective theory of mind in Parkinson's disease. Neuropsychiatric Disease and Treatment. 2013;9:1071.

38.Dehaene S, Nakamura K, Jobert A, Kuroki C, Ogawa S, Cohen L. Why do children make mirror errors in reading? Neural correlates of mirror invariance in the visual word form area. Neuroimage. 2010 Jan 15;49(2):1837-48.

39.Gallese V, Lakoff G. The brain's concepts: The role of the sensory-motor system in conceptual knowledge. Cognitive Neuropsychology. 2005 May 1;22(3-4):455-79. 
40.Eisen A, Turner MR, Lemon R. Tools and talk: an evolutionary perspective on the functional deficits associated with amyotrophic lateral sclerosis. Muscle \& Nerve. 2014 Apr;49(4):469-77.

41.Bede P, Iyer PM, Schuster C, Elamin M, Mclaughlin RL, Kenna K, Hardiman O. The selective anatomical vulnerability of ALS:'disease-defining' and 'diseasedefying'brain regions. Amyotrophic Lateral Sclerosis and Frontotemporal Degeneration. 2016 Nov 16;17(7-8):561-70.

42.Bede P, Omer T, Finegan E, Chipika RH, Iyer PM, Doherty MA, Vajda A, Pender N, McLaughlin RL, Hutchinson S, Hardiman O. Connectivity-based characterisation of subcortical grey matter pathology in frontotemporal dementia and ALS: a multimodal neuroimaging study. Brain Imaging and Behavior. 2018 Dec 1;12(6):1696-707.

43.Alruwaili AR, Pannek K, Coulthard A, Henderson R, Kurniawan ND, McCombe P. A combined tract-based spatial statistics and voxel-based morphometry study of the first MRI scan after diagnosis of amyotrophic lateral sclerosis with subgroup analysis. Journal of Neuroradiology. 2018 Feb 1;45(1):41-8.

44.Turner MR, Swash M. The expanding syndrome of amyotrophic lateral sclerosis: a clinical and molecular odyssey. Journal of Neurology, Neurosurgery \& Psychiatry. 2015 Jun 1;86(6):667-73.

45.Trojsi F, Monsurro MR, Esposito F, Tedeschi G. Widespread structural and functional connectivity changes in amyotrophic lateral sclerosis: insights from advanced neuroimaging research. Neural Plasticity. 2012 Jan 1;2012. 